Bull. Austral. Math. Soc.

$53 \mathrm{c} 40,53 \mathrm{c} 20,53 \mathrm{c} 42$

VoL. $62(2000) \quad[165-170]$

\title{
A SHARP LOWER BOUND FOR THE RICCI CURVATURE OF BOUNDED HYPERSURFACES IN SPACE FORMS
}

\author{
Alain R. Veeravalli \\ Dedicated to LAmiae and LuCAS ZaKaria With GREAT AfFection.
}

We give a sharp lower bound for the Ricci curvature of bounded complete hypersurfaces of space forms. This leads to several applications.

\section{INTRODUCTION AND THE MAIN RESULT.}

It is easy to see that if a closed smooth plane curve is included in a disk of radius $r>0$, then there exists a point of the curve for which the curvature is in absolute value greater than or equal to $1 / r$. A similar result holds for surfaces: any compact surface of $\mathbb{R}^{3}$ included in a ball of radius $r$ admits a point for which the Gaussian curvature is greater than or equal to $1 / r^{2}$. In 1983 , Leung extended these results by showing the following.

THEOREM. [4] If $M$ is a complete hypersurface of $\mathbb{R}^{n+1}(n \geqslant 2)$ included in a ball of radius $r>0$ with sectional curvature bounded away from $-\infty$, then

$$
\limsup _{\xi \in U M} \operatorname{Ric}(\xi, \xi) \geqslant \frac{n-1}{r^{2}}
$$

where Ric is the Ricci curvature of $M$ and $U M$ the unit tangent bundle of $M$.

Note that for the sphere of radius $r$ in $\mathbb{R}^{n+1}$ the above inequality is in fact an equality. A natural question is to search for the Ricci curvature lower bound when replacing the Euclidean space by any space form. In two recent papers, Beltagy [1] and Erdŏgan [3] tried to give an answer but infortunately some estimates are false and the others are not sharp. In a previous paper [8], the author dealt with a close problem whose ideas can be used to settle the question. The main result of this work is the following:

THEOREM. Let $\tilde{S}_{n+1}(c)$ be the simply connected space form of constant sectional curvature $c(c \in \mathbb{R}, n \geqslant 2), M$ a complete hypersurface of $\tilde{S}_{n+1}(c)$ with sectional curvature

Received 22nd December, 1999

Copyright Clearance Centre, Inc. Serial-fee code: 0004-9727/00 \$A2.00+0.00. 
bounded away from $-\infty$ and included in a closed normal ball of radius $r>0$ in $\tilde{S}_{n+1}(c)$, with $r<\pi /(2 \sqrt{c})$ if $c>0$. Then

$$
\underset{\xi \in U M}{\limsup } \operatorname{Ric}(\xi, \xi) \geqslant(n-1)\left(c+k_{c}^{2}(r)\right)
$$

where Ric is the Ricci curvature of $M, U M$ the unit tangent bundle of $M$ and

$$
k_{c}(r)=\left\{\begin{array}{cl}
\sqrt{c} \cot (r \sqrt{c}) & \text { if } c>0 \\
1 / r & \text { if } c=0 \\
\sqrt{-c} \operatorname{coth}(r \sqrt{-c}) & \text { if } c<0
\end{array}\right.
$$

As one can see, this generalises Leung's theorem. Moreover, the function $k_{c}$ is well-known by Riemannian geometers: the distance sphere of radius $r$ in $\tilde{S}_{n+1}(c)$, with $r<\pi / \sqrt{c}$ if $c>0$, is an umbilical hypersurface with principal curvatures being precisely $k_{c}(r)$. It also shows by the Gauss formula that its Ricci curvature is constant and equal to $(n-1)\left(c+k_{c}^{2}(r)\right)$. Therefore the inequality given in our theorem is sharp.

Before giving the proof, one can remark that $(n-1)\left(c+k_{c}^{2}(r)\right)$ is positive for any constant $c$ and positive $r$ (with $r<\pi / \sqrt{c}$ if $c>0$ ). This leads to criteria of unboundness:

COROLLARY 1. Let $M$ be a complete hypersurface of $\tilde{S}_{n+1}(c)(n \geqslant 2)$ with sectional curvature bounded away from $-\infty$ and nonpositive Ricci curvature. If $c \leqslant 0$, then $M$ is unbounded. If $c>0$, then the diameter of $M$ (for both Riemannian distances on $M$ and $\left.\tilde{S}_{n+1}(c)\right)$ satisfies $\operatorname{diam}(M) \geqslant \pi /(2 \sqrt{c})$.

When $M$ is compact, these results can be reformulated in an easier way:

\section{Corollary 2 .}

(i) Let $M$ be a compact hypersurface of $\tilde{S}_{n+1}(c)$. If $c$ is nonpositive, then there exists a point $q \in M$ and a unit tangent vector $u$ to $M$ at $q$ such that

$$
\underset{q}{\operatorname{Ric}}(u, u) \geqslant(n-1)\left(c+k_{c}^{2}(r)\right)(>0)
$$

where $r$ is the radius of any ball in $\widetilde{S}_{n+1}(c)$ containing $M$. Therefore, if $c$ is nonpositive, there is no compact hypersurfaces in $\tilde{S}_{n+1}(c)$ with nonpositive Ricci curvature. In particular, if $c$ is nonpositive, there is no compact minimal hypersurfaces in $\widetilde{S}_{n+1}(c)$.

(ii) If $c$ is positive, then the diameter of any compact hypersurface $M$ of $\tilde{S}_{n+1}(c)$ with nonpositive Ricci curvature satisfies $\operatorname{diam}(M) \geqslant \pi /(2 \sqrt{c})$.

\section{Preliminary RESUlts.}

Let $(\widetilde{M},\langle\rangle$,$) be a Riemannian manifold, \widetilde{\nabla}$ its Levi-Civita connection and $\tilde{f}: \widetilde{M} \rightarrow$ $\mathbb{R}$ a smooth function on $\widetilde{M}$. Recall that the gradient of $\tilde{f}$ is a smooth vector field $\widetilde{\nabla} \tilde{f}$ 
on $\widetilde{M}$ defined by $\langle\widetilde{\nabla} \tilde{f}, X\rangle=X \tilde{f}$ and the Hessian of $\tilde{f}$ is the (0,2)-symmetric tensor $\widetilde{\nabla}^{2} \tilde{f}$ defined by $\widetilde{\nabla}^{2} \tilde{f}(X, Y)=\left\langle\widetilde{\nabla}_{X}(\widetilde{\nabla} \tilde{f}), Y\right\rangle=\widetilde{X}(\tilde{Y} \tilde{f})-\left(\widetilde{\nabla}_{X} Y\right) \tilde{f}, X$ and $Y$ being smooth vectors fields on $\widetilde{M}$. If $M$ is a submanifold of $\widetilde{M}$ with the induced metric and the induced connection $\nabla$, we can also define the gradient $\nabla f$ and the Hessian $\nabla^{2} f$ of any smooth function $f$ on $M$. For the particular case of smooth functions $f$ on $M$ of the form $f=\tilde{f} \circ i$ (where $i: M \rightarrow \widetilde{M}$ is the canonical injection), these operators are related: for any vector fields $X, Y$ on $M$, we have

$$
\begin{aligned}
\widetilde{\nabla}^{2} \tilde{f}(X, Y) & =X(Y \tilde{f})-\left(\widetilde{\nabla}_{X} Y\right) \tilde{f} \\
& =X(Y \tilde{f})-\left\{\left(\nabla_{X} Y\right)+S(X, Y)\right\} f \\
& =\nabla^{2} f(X, Y)-\langle\widetilde{\nabla} \tilde{f}, S(X, Y)\rangle
\end{aligned}
$$

where $S$ is the second fundamental form of $M$.

The gradient and Hessian are used in the classical Hopf lemma which says that for a smooth function $f: M \rightarrow \mathbb{R}$ on a compact Riemannian manifold $M$, there exists a point $q \in M$ such that $\nabla f(q)=0$ and $\nabla^{2} f(q)(X, X) \leqslant 0$ for any vector $X \in T_{q} M$. The proof of our theorem uses a rather technical result due to Omori which can be seen as a generalisation of the Hopf lemma:

THEOREM [5] Let $M$ be a complete Riemannian manifold with sectional curvature bounded away from $-\infty$ and $f: M \rightarrow \mathbb{R}$ a smooth function on $M$ bounded from above. Then for any $q_{0} \in M$ and any $\varepsilon>0$, there exists a point $q \in M$ such that $f(q) \geqslant f\left(q_{0}\right)$, $|\nabla f(q)|<\varepsilon$ and $\nabla^{2} f(q)(X, X)<\varepsilon$ for any unit tangent vector $X$ at $q$.

Another trick used in the proof is an algebraic lemma due to Otsuki:

LEMma [7] Let $S: \mathbb{R}^{n} \times \mathbb{R}^{n} \rightarrow \mathbb{R}^{k}$ be a symmetric bilinear form on $\mathbb{R}^{n}(n, k>0)$. If $S^{n-1}$ denotes the unit sphere of $\mathbb{R}^{n}$, then the function $S^{n-1} \rightarrow \mathbb{R}: x \mapsto|S(x, x)|^{2}$ achieves its minimum at a point $x_{0}$ and we have the following properties

(i) $x_{0} \perp \operatorname{Ker} S\left(x_{0}, \cdot\right)$

(ii) $\left\langle S\left(x_{0}, x_{0}\right), S(x, x)\right\rangle \geqslant\left|S\left(x_{0}, x_{0}\right)\right|^{2}$ for any unit vector $x \in \operatorname{Ker} S\left(x_{0}, \cdot\right)$.

At last, the crucial point in the proof is the following result which uses classical material of Riemannian geometry:

Proposition. Let $(\widetilde{M},\langle\rangle$,$) be a Riemannian manifold, p$ a point of $\widetilde{M}, d$ the Riemannian distance of $\widetilde{M}$ and $\tilde{f}: \widetilde{M} \rightarrow \mathbb{R}: q \mapsto d_{p}^{2}(q) / 2$. Then

(i) $\tilde{f}$ is smooth on $\widetilde{M} \backslash \operatorname{Cut}(p)$ where $\operatorname{Cut}(p)$ is the cut point of $p$.

(ii) For any $q \in \widetilde{M}$ and any tangent vector $v$ to $\widetilde{M}$ at $q$, we have $\widetilde{\nabla} \tilde{f}(q)$ $=d_{p}(q) \dot{\gamma}\left(d_{p}(q)\right)$ and $\widetilde{\nabla}^{2} \tilde{f}(q)(v, v)=d_{p}(q)\left\langle\widetilde{\nabla} X\left(d_{p}(q)\right), X\left(d_{p}(q)\right)\right\rangle$ where $\gamma:\left[0, d_{p}(q)\right] \rightarrow \widetilde{M}$ is the unique normal geodesic joining $p$ to $q, X$ the unique Jacobi field along $\gamma$ with the boundary condition $\left(X(0), X\left(d_{p}(q)\right)\right)$ $=(0, v)$ and $\widetilde{\nabla} X$ the covariant derivative of $X$ along $\gamma$. 
(iii) In particular, if $\widetilde{M}=\widetilde{S}_{n+1}$ (c) is a space form of constant sectional curvature $c$ and $p$ is a point of $\widetilde{M}$, then for any $q \in B_{p}(r)$, with $r<\pi /(2 \sqrt{c})$ if $c>0$, and any tangent vector $v$ to $\widetilde{M}$ at $q$ with $v \perp \widetilde{\nabla} d_{p}(q)$, we have $\nabla^{2} \widetilde{f}(q)(v, v)$ $=d_{p}(q) \cdot k_{c}\left(d_{p}(q)\right) \cdot|v|^{2}$.

The first two points come from the first and second variation formulae for length and energy (see, for example, [2] or [6]). For the last point, it suffices to solve the differential equation satisfied by the Jacobi field.

\section{Proof of the theorem.}

The manifold $M$ is endowed with the metric $\langle$,$\rangle induced by \widetilde{S}_{n+1}(c)$. The Riemannian distance in $\tilde{S}_{n+1}(c)$ will be noted $d$ and $U M=\bigcup_{q \in M} U_{q} M$ will mean the unit tangent bundle of $M$. We shall also consider the function $\tilde{f}=d_{p}^{2} / 2: \widetilde{S}_{n+1}(c) \rightarrow \mathbb{R}$ and its restriction $f=d_{p}^{2} / 2: M \rightarrow \mathbb{R}$. As $M$ is included in a closed normal ball $\bar{B}_{p}(r)$, the manifold $M$ avoids the cut point of $p$ in $\widetilde{S}_{n+1}(c)$ and therefore the function $f$ is smooth (by the above proposition) and bounded by $r^{2} / 2$. Choose a point $q_{0}$ in $M$ different from $p$. By Omori's theorem, for any positive integer $m$, there exists a point $q_{m} \in M$ such that $f\left(q_{m}\right) \geqslant f\left(q_{0}\right),\left|\nabla f\left(q_{m}\right)\right|<1 / m$ and $\nabla^{2} f\left(q_{m}\right)(u, u)<1 / m$ for any $u \in U_{q_{m}} M$. Remark that $0<d_{p}\left(q_{0}\right) \leqslant d_{p}\left(q_{m}\right) \leqslant r$. For any integer $m \geqslant 0$, we shall write for convenience $\ell_{m}$ for $d_{p}\left(q_{m}\right)$ and $\gamma_{m}$ for the unique normal geodesic joining $p$ to $q_{m}$. Fix now a positive integer $m$ and a vector $u \in U_{q_{m}} M$. By Omori's theorem, equation (1), the previous proposition and the Cauchy-Schwarz inequality, we have

$$
\begin{aligned}
1 / m>\nabla^{2} f\left(q_{m}\right)(u, u) & =\widetilde{\nabla}^{2} \tilde{f}\left(q_{m}\right)(u, u)+\ell_{m}\left\langle\dot{\gamma}_{m}\left(\ell_{m}\right), S_{q_{m}}(u, u)\right\rangle \\
& \geqslant \widetilde{\nabla}^{2} \tilde{f}\left(q_{m}\right)(u, u)-\ell_{m} \cdot\left|S_{q_{m}}(u, u)\right| .
\end{aligned}
$$

Our next work will be to estimate the first term of the right-hand side of equation (2). We remark that $u$ need not to be normal to $\widetilde{\nabla} \tilde{f}\left(q_{m}\right)\left(=\ell_{m} \dot{\gamma}_{m}\left(\ell_{m}\right)\right)$. In order to apply the preceding proposition, share $u$ in two parts: $u=u^{t}+u^{n}$ where $u^{t}$ (respectively $u^{n}$ ) is normal (respectively parallel) to $\widetilde{\nabla} \tilde{f}\left(q_{m}\right)$. Then

$$
\widetilde{\nabla}^{2} \tilde{f}\left(q_{m}\right)(u, u)=\widetilde{\nabla}^{2} \tilde{f}\left(q_{m}\right)\left(u^{t}, u^{t}\right)+2 \cdot \widetilde{\nabla}^{2} \tilde{f}\left(q_{m}\right)\left(u^{t}, u^{n}\right)+\widetilde{\nabla}^{2} \tilde{f}\left(q_{m}\right)\left(u^{n}, u^{n}\right) .
$$

Of course, we have by the above proposition

$$
\widetilde{\nabla}^{2} \tilde{f}\left(q_{m}\right)\left(u^{t}, u^{t}\right)=\ell_{m} \cdot k_{c}\left(\ell_{m}\right) \cdot\left|u^{t}\right|^{2} .
$$

Since $u^{n}=\left\langle u, \widetilde{\nabla} \tilde{f}\left(q_{m}\right)\right\rangle \dot{\gamma}_{m}\left(\ell_{m}\right) / \ell_{m}$ and since $\left\langle u, \widetilde{\nabla} \tilde{f}\left(q_{m}\right)\right\rangle=\left\langle u, \nabla f\left(q_{m}\right)\right\rangle$, we obtain by Omori's theorem and the Cauchy-Schwarz inequality

$$
\left|u^{n}\right|<\frac{1}{m \ell_{m}} \leqslant \frac{1}{m \ell_{0}}
$$


and so

$$
\left|u^{t}\right|>1-\frac{1}{m \ell_{m}} \geqslant 1-\frac{1}{m \ell_{0}} .
$$

From now, we shall assume that $m$ is choosen sufficiently large to ensure that $1-\left(1 / m \ell_{0}\right)>0$.

Finally, note that the linear map $L_{q_{m}}: T_{q_{m}} \widetilde{S}_{n+1}(c) \rightarrow T_{q_{m}} \widetilde{S}_{n+1}(c): w \mapsto \widetilde{\nabla}_{w}(\widetilde{\nabla} \tilde{f})$ is continuous. So $\left\|L_{q_{m}}\right\|<\infty$ and we can write that for any tangent vectors $w_{1}$ and $w_{2}$ to $\tilde{S}_{n+1}(c)$ at $q_{m}$,

$$
\left|\widetilde{\nabla}^{2} \tilde{f}\left(q_{m}\right)\left(w_{1}, w_{2}\right)\right|=\left|\left\langle L_{q_{m}}\left(w_{1}\right), w_{2}\right\rangle\right| \leqslant\left\|L_{q_{m}}\right\| \cdot\left|w_{1}\right| \cdot\left|w_{2}\right| .
$$

By using the continuity of the map $\bar{B}_{p}(r) \rightarrow \mathbb{R}: q \mapsto\left\|L_{q}\right\|$ on the compact set $\bar{B}_{p}(r)$, there exists a positive constant $a$ such that on $\bar{B}_{p}(r)$,

$$
\left|\widetilde{\nabla}^{2} \tilde{f}(q)\left(w_{1}, w_{2}\right)\right| \leqslant a \cdot\left|w_{1}\right| \cdot\left|w_{2}\right| \text {. }
$$

Combining inequalities (3), (4), (5), (6), $\left|u^{t}\right| \leqslant 1,\left|u^{n}\right| \leqslant 1$ and since $k_{c}$ is a decreasing function, we obtain

$$
\begin{aligned}
\bar{\nabla}^{2} \tilde{f}\left(q_{m}\right)(u, u) & \geqslant \ell_{m} \cdot k_{c}\left(\ell_{m}\right) \cdot\left|u^{t}\right|^{2}-2 a\left|u^{t}\right| \cdot\left|u^{n}\right|-a\left|u^{n}\right|^{2} \\
& \geqslant \ell_{m} \cdot k_{c}(r)\left\{1-\frac{1}{m \ell_{0}}\right\}^{2}-3 a\left|u^{n}\right| \\
& >\ell_{m} \cdot k_{c}(r)\left\{1-\frac{1}{m \ell_{0}}\right\}^{2}-\frac{3 a}{m \ell_{0}}
\end{aligned}
$$

By inequality (2), we conclude that

$$
\left|S_{q_{m}}(u, u)\right|>k_{c}(r)\left\{1-\frac{1}{m \ell_{0}}\right\}^{2}-\frac{3 a}{m \ell_{0}^{2}}-\frac{1}{m \ell_{0}} .
$$

Since $k_{c}$ is a positive function, one sees that, for sufficiently large $m,\left|S_{q_{m}}(u, u)\right|$ is positive.

Among all vectors of $U_{q_{m}} M$, let $u_{1}$ be one which makes $\left|S_{q_{m}}\right|$ minimal on the diagonal of $U_{q_{m}} M$. By the last remark, $\left|S_{q_{m}}\left(u_{1}, u_{1}\right)\right|>0$ for sufficiently large $m$ and so the kernel of the linear map $S_{q_{m}}\left(u_{1}, \cdot\right): T_{q_{m}} M \rightarrow\left(T_{q_{m}} M\right)^{\perp}$ is $(n-1)$-dimensional. If $\left\{u_{2}, \ldots, u_{n}\right\}$ is an orthonormal basis of this kernel, the first part of Otsuki's lemma asserts that $\left\{u_{1}, \ldots, u_{n}\right\}$ is an orthonormal basis of $T_{q_{\mathrm{m}}} M$. By the above inequality, the Gauss formula and the second part of Otsuki's lemma, we have

$$
\begin{aligned}
\underset{q_{m}}{\operatorname{Ric}}\left(u_{1}\right) & =(n-1) c+\sum_{i=2}^{n}\left\langle S_{q_{m}}\left(u_{1}, u_{1}\right), S_{q}\left(u_{i}, u_{i}\right)\right\rangle \\
& \geqslant(n-1) c+\sum_{i=2}^{n}\left|S_{q_{m}}\left(u_{1}, u_{1}\right)\right|^{2} \\
& \geqslant(n-1) c+(n-1)\left\{k_{c}(r)\left\{1-\frac{1}{m \ell_{0}}\right\}^{2}-\frac{3 a}{m \ell_{0}^{2}}-\frac{1}{m \ell_{0}}\right\}^{2} .
\end{aligned}
$$

By letting $m$ going to $+\infty$, this leads to the announced inequality. 


\section{REMARKS}

1. It was asserted in [3] without proof that for the elliptic space form $S^{n+1}=\tilde{S}_{n+1}(1)$, we have

$$
\limsup _{\xi \in U M} \operatorname{Ric}(\xi, \xi) \geqslant(n-1)\left(1+\cos ^{4}(r / 2) / \sin ^{2}(r / 2)\right) .
$$

An easy computation shows that

$$
(n-1)\left(1+k_{1}^{2}(r)\right)-(n-1)\left(1+\cos ^{4}(r / 2) / \sin ^{2}(r / 2)\right)<0
$$

for any positive $r<\pi / 2$. So, the assertion in [3] cannot be true as was shown above by taking the hypersphere. For the hyperbolic space form $\mathbb{H}^{n+1}=\widetilde{S}_{n+1}(-1)$, our result sharpens the inequality given in [3].

2. If $M$ is assumed to be compact, the proof can be shortened of course by using the Hopf lemma.

\section{REFERENCES}

[1] M. Beltagy, 'A study of Ricci curvature of space forms', Atti Accad. Sci. Torino Cl. Sci. Fis. Mat. Natur. 126 (1992), 177-185.

[2] J. Cheeger and D.G. Ebin, Comparison theorems in Riemannian geometry (North-Holland, Amsterdam, 1975).

[3] M. Erdŏgan, 'On the Ricci curvature of a hypersurface in a space form', Geom. Dedicata 61 (1996), 221-225.

[4] P.F. Leung, 'Complete hypersurface of non-positive Ricci curvature', Bull. Austral. Math. Soc. 27 (1983), 215-219.

[5] H. Omori, 'Isometric immersions of Riemannian manifolds', J. Math. Soc. Japan 19 (1967), 205-214.

[6] T. Sakai, Riemannian geometry, Translations of Mathematical Monographs 149 (Amer. Math. Soc., Providence, R.I., 1996).

[7] M. Spivak, A comprehensive introduction to differential geometry 5 (Publish or Perish, Boston, Mass., 1975).

[8] A.R. Veeravalli, 'A rigidity theorem for compact hypersurfaces with an upper bound for the Ricci curvature', Geometriae Dedicata 74 (1999), 287-290.

Département de Mathématiques

Université d'Evry-Val d'Essonne

Boulevard des Coquibus

91025 Evry Cedex

France

e-mail: Alain.Veeravalli@maths.univ-evry.fr 